\title{
Should acetylsalicylic acid (ASA) therapy for prevention of thromboembolic events be stopped prior to surgical extractions?
}

\author{
Abstracted from \\ Medeiros FB, de Andrade AC, Angelis GA, et al. \\ Bleeding evaluation during single tooth extraction in patients with coronary artery disease \\ and acetylsalicylic acid therapy suspension: a prospective, double-blinded, and randomized study. \\ J Oral Maxillofac Surg 2011; 69: 2949-2955. Epub 2011 Jul 29. PubMed PMID: 21802823. \\ Address for correspondence: Dr Medeiros, Department of Dentistry, \\ Sociedade Paulista de Cardiologia do Estado, São Paulo, Brazil; E-mail: fredericobuhatem@yahoo.com.br
}

Question: In patients with coronary artery

disease on acetylsalicylic acid (ASA) therapy

requiring dental extraction does stopping ASA

affect degree of bleeding?

\section{Design Randomised controlled trial}

Intervention Patients with coronary artery disease who were receiving $100 \mathrm{mg} /$ day of ASA for the prevention of thromboembolic events, and requiring at least one molar tooth extracted were randomised to either having their ASA therapy suspended for seven days before tooth extraction and restarted the day following the surgical procedure or not having their ASA therapy suspended at any point before or after the procedure. A single dentist who was unaware of the patients' ASA therapy status performed all the extractions.

Outcome measure Outcomes were a platelet aggregation test carried out on the day of the operation and the amount of bleeding measured during the intra-operative period.

Results Bleeding was controlled with local haemostatic methods and there were no reported episodes of haemorrhaging during the intraand post-operative periods. The mean $( \pm S D)$ volume of bleeding was $12.10 \pm 9.37 \mathrm{~mL}$ for patients who underwent ASA therapy suspension and $16.38 \pm 13.54 \mathrm{~mL}$ for those patients whose treatments were unaltered $(P=.151)$. The platelet reactivity index values exhibited statistically significant differences between the two investigated groups $(\mathrm{P}=.004)$. The platelet reactivity index values for the group with ASA therapy suspended was $242.58 \pm 71.26$ compared with $192.09 \pm 60.54$ in the group that continued with ASA.

Conclusions There was no difference in the amount of bleeding that occurred during tooth extraction between patients who continued ASA therapy and patients who suspended their ASA therapy. The platelet reactivity test demonstrated a reduction in platelet aggregation in the ASA therapy group, but this was without clinical consequence.

\section{Commentary}

The purpose of this paper was to address the following clinical question: Among patients with coronary artery disease (CAD) taking low dose (100 mg each day) acetylsalicylic acid (ASA) and need more than one molar removed, do those who stop ASA seven days preoperatively and then restart postoperatively, when compared to those who do not stop ASA therapy, have a decreased risk for postoperative bleeding?

To address the research question, the investigators implemented a double blind, randomised clinical trial (RCT). The primary predictor variable was the treatment group (ASA suspension - yes or no). The primary outcome variable was postoperative estimated blood loss (EBL).

There was mean difference in EBL of $4 \mathrm{cc}$ between the two groups favouring the group stopping ASA. The key conclusion of the paper was that there was no clinically or statistically significant difference in postoperative EBL between the two groups, and stopping ASA therapy before extractions is not indicated.

This paper adds to the body of literature suggesting that stopping ASA therapy is not indicated prior to tooth extraction. There are a few study design issues to address:

1. Blinding

2. Duration of stopping ASA and

3. EBL as an outcome variable.

This paper is 'advertised' as a double-blind study. While the surgeon and the analytic team were blind to the treatment, I do not think the patients were blinded. One group continued its ASA therapy and the other group suspended ASA treatment for a week. I did not see evidence that the group who stopped ASA therapy continued on a placebo dose. I do not think this is a major flaw; however, when the investigators are not sure of their study design, it casts a long shadow on the overall quality of the study.

The duration of discontinuing ASA may not have been long enough, ie seven days, to achieve the desired therapeutic effect. Platelets survive about ten days. If the investigators discontinued ASA for seven days, there was a persistent sub-population of platelets present with abnormal function. As such, the investigators may have underestimated the treatment effect of discontinuing ASA preoperatively.

EBL is a notoriously difficult parameter to estimate. While the authors measured intra-operative blood loss well, how did they ascertain post-operative blood loss? Additionally, I am not sure intra-operative blood loss was the best parameter to measure 
because it is usually nominal. With anticoagulated patients, I rarely have intra-operative bleeding problems. It is in the following two to four days when the patients return reporting persistent bleeding. Rather than EBL, I think it would be far more valuable to estimate patient-centred outcomes such as infection, possibly due to retained clotting promoters inserted into extraction sockets, number of visits to the clinic or emergency department for management of persistent postoperative bleeding, transfusion or hospital admission.
Tom Dodson

Department of Oral and Maxillofacial Surgery, Harvard School of Dental Medicine, Boston, MA; Director, Center for Applied Clinical Investigation and Attending Oral and Maxillofacial Surgeon, Department of Oral and Maxillofacial Surgery, Massachusetts General Hospital, Boston, Massachusetts, USA.

Evidence-Based Dentistry (2012) 13, 89-90. doi:10.1038/sj.ebd.6400881 